RESEARCH ARTICLE

\title{
Can Cochlear Implantation Improve Voice in Speaking Children?
}

\author{
Rabeea A El Sabeela ${ }^{1}$ and Safinaz N Azab, MD ${ }^{2 *}$ \\ ${ }^{1}$ Department of Otorhinolaryngology, King Abdel Aziz Specialized Hospital, Kingdome of Saudi Arabia \\ ${ }^{2}$ Department of Otorhinolaryngology, Faculty of Medicine, Beni-Suef University, Egypt
}

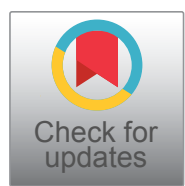

*Corresponding author: Safinaz N Azab, MD, Assistant Professor of Phoniatrics, Department of Otorhinolaryngology, Faculty of Medicine, Beni-Suef University, Egypt, Tel: 02-26076976; 01007838481

\begin{abstract}
Objective: Some of voice characteristics of deaf people differ considerably from those with normal hearing. After cochlear implantation, auditory control of voice production is possible and quality of voice is improved.

Aim: The aim of this study was to investigate changes in some of voice parameters in deaf children after cochlear implantation.

Methods: Thirty prelingually deafened children implanted unilaterally at the age of 3-6 years were included in the study. For all of the children an acoustic analysis of Arabic vowel /æ/ was performed before cochlear implantation, 6, and 12 months after the implantation. Fundamental frequency (f0), jitter, shimmer and noise-to-harmonic ratio (NHR) were compared before and after implantation. Results of acoustic analyses were compared for children who were implanted before or at the age of 4 years and children who were implanted after the age of 4 years.
\end{abstract}

Results: After cochlear implantation fundamental frequency did not change significantly. However, an improvement was noticed in measurements of jitter $(p=0.006)$ and shimmer $(p=0.021)$ as early as 6 months after the implantation. The noise-to-harmonic ratio improved $(p=0.010) 12$ months after implantation. The children implanted before or at the age of 4 years showed a significant improvement in jitter $(p=$ $0.003)$ and shimmer $(p=0.004)$ as early as 6 months noiseto harmonic ratio $(p=0.021) 12$ months after implantation. In children implanted after the age of 4 years the significant changes was detected in fO $(p=0.045), 12$ months after the implantation and in Shimmer $(p=0.017)$, also 12 months after the implantation.

Conclusion: The results of the present study have confirmed that cochlear implantation enables auditory moment-tomoment control of pitch and loudness. The determination of jitter and shimmer in the vowel Arabic /æ/ sample proved to be a good and early indicator of improved phonation control, even in young children. The deaf children who were implanted before the age of 4 years improved their voice quality and control more quickly and to a greater extent than the children implanted after the age of 4 years.

\section{Introduction}

AJ Yates [1] has described the feedback information for speech as coming from three sources: The kinesthetic and proprioceptive feedback from the muscular and sensory apparatus involved in speaking, and auditory control. Auditory feedback affects both the moment-to-moment and the late control of speech. Moment-to-moment auditory feedback is important for the control of the suprasegmental characteristics of voice and speech, such as fundamental frequency (fO) and voice intensity and quality. It has-been suggested that auditory feedback also influences the control of respiratory, phonatory, and articulatory functions [25]. There is general agreement in the literature that some of the voice characteristics of deaf people differ considerably from those of speakers with normal hearing. Congenitally deaf speakers tend to have a higher f0 than speakers with normal hearing [6-8]. On the other hand, some investigators did not find a higher fo in young hearing impaired children than they found in the controls [9]. Lack of auditory control also affects the control of voice intensity, Leder, et al. [10] suggested that adventitious profound deafness was associated with a significantly increased voiceintensity level and greater fluctuations in intensity production. After cochlear implantation the auditory control of voice and speech is possible, and there are some reports of voice and speech improvement after implantation. Most of the investigators measured fo and the variability of pitch and intensity in spontaneous talking or during the reading of a standard text. Ball and Ison [11] found in two of their patients that vocal-fold activity became more regular after implantation. Leder, et al. [12] noted that when adequate auditory feedback is provided after implantation fo was found to be one 
of the earliest acoustic characteristics to come close to normal values again. Smoorenburg, et al. [13] compared speech samples before and 1-4 years after implantation in 12 subjects. Abnormally high pitches moved partially toward normal values after the implantation as did abnormally large pitch modulation. Hamzavi, et al. [14] evaluated the short-term effect of a cochlear implantation on f0. The acoustic analysis of voice recordings performed before and 3 months after the implantation showed that $38 \%$ of the studied 13 patients had a significant decrease of $\mathrm{fO}$ after the implantation. A post-operative decrease of fo was generally observed in all patients. Higgins, et al. [8] studied the speech/voice physiological characteristics of prelingually deafened children before and after cochlear implantation. The children who received an implant after 5 years of age and were educated in a verbal communication showed persistence and further development of deviant speech/ voice behaviors for several years after the implant surgery. Monini, et al. [15] evaluated the moment-tomoment auditory control of voice at an early stage after cochlear implantation in three children. The fo in the voice samples of the Italian vowel 'a' remained constant in one child, increased in one child, and decreased in one child from the presurgical situation to the implant activated situation immediately after the first fitting session. Seifert, et al. [16] Used Kay CSL 4300B to investigate the $\mathrm{f} 0$ in the German vowel ' $a$ ' at different time points after the cochlear implantation in 20 prelingually deafened children. Children who had been operated on before their fourth birthday showed no significant difference in their fo from their age- and sexmatched peers. However, a significant difference in fo was documented for the children who were older at the time of implantation. The investigators concluded that prelingually deafened children who receive a cochlear implant before their fourth birthday attain a better auditory control over their voice and speech.

\section{Aim of the Study}

The aim of the current study is to assess the influence of auditory control on acoustic voice parameters in prelingually deafened children after cochlear implantation and hence, improvement of voice quality of the implanted Arabic speaking children.

\section{Methods}

Thirty prelingually deafened children (15 boys, 15 girls) were included in the study. The children received a cochlear implant at the age of 2.5-6 years (mean 3.24 years, standard deviation 0.65 years). Fifteen children were implanted before or at the age of 4 years and 15 children were implanted after the age of 4 years. They were all implanted with a multi-channel $\mathrm{Cl}$. After the implantation the average values on a pure tone audiogram for the frequencies 500, 1000, 2000 and $4000 \mathrm{~Hz}$ ranged from 21.25 to $46.75 \mathrm{~dB}$ (mean 31.99 $\mathrm{dB}$, standard deviation $5.78 \mathrm{~dB}$ ). All the children had hearing training, language, and speech therapy after the implantation. The voice samples of a sustained Arabic vowel /æ/ at a habitual pitch and loudness, for duration of 3s, were analyzed with a Multi-Dimensional Voice Program (Kay Elemetrics, USA). The vowel was chosen because it can be produced even by young children and is mainly dependent on acoustic rather than or sensitive control [17]. The average fundamental frequency (f0), jitter (JIT), shimmer (SH) and noise-to-harmonic ratio (NHR) were determined for every voice sample. JIT gives an evaluation of the short-term variability of the pitch period. SH gives an evaluation of the short-term variability of the peak-to-peak amplitude (loudness) within the analyzed voice sample. NHR is an average ratio of the energy of the inharmonic components in the range $1500-4500 \mathrm{~Hz}$ to the harmonic components' energy in the range $70-4500 \mathrm{HZ}$, and represents a general evaluation of noise presence in the analyzed signal. According to the recommendation of the European Laryngological Society, JIT and SH are used to estimate phonation quality [18].

The voice samples were analyzed before the implantation, 6 and 12 months after the implantation. In the case of acute respiratory infection influencing the phonation, the child was invited for a voice-samples analysis 3 weeks later. During every session, three live voice samples were analyzed. The mean values for fo, $\mathrm{JIT}, \mathrm{SH}$, and NHR were considered for further evaluation. The results of the acoustic analysis of the voice samples before the implantation were compared to the voice samples 6 , and 12 , months after the implantation. The results of the acoustic analysis were compared for the children who were implanted before or at the age of 4 years and the children who had received their $\mathrm{Cl}$ after the age of 4 years. Paired and unpaired t-test of the statistical package SPSS, version 11.0, were used.

Table 1: Results of the acoustic analysis of the vowel /æ/ samples performed before and 6 months after cochlear implantation in deaf children $(\mathrm{N}=30)$.

\begin{tabular}{|l|l|l|l|}
\hline Acoustic parameter & Before $\mathbf{C l}$ (mean/S.D.) & $\mathbf{6}$ months after Cl (mean/S.D.) & p \\
\hline fO $(\mathrm{Hz})$ & $286.00 / 58.22$ & $295.52 / 57.09$ & 0.514 \\
\hline JIT $(\%)$ & $2.89 / 1.87$ & $1.75 / 1.312$ & $\mathbf{0 . 0 0 6}$ \\
\hline SH (\%) & $6.27 / 3.78$ & $4.39 / 1.80$ & $\mathbf{0 . 0 2 1}$ \\
\hline NHR & $0.18 / 0.11$ & $0.18 / 0.23$ & 0.925 \\
\hline
\end{tabular}

Bold values indicate statistical significance. 


\section{Results}

The results of the acoustic analysis of the voice samples detected a significant improvement in JIT, SH, and NHR after the implantation (Tables 1 and Table 2). When the results of the acoustic analysis in the children who were implanted before or at the age of 4 years were compared with the results from the children

Table 2: Results of the acoustic analysis of the vowel /æ/ samples performed before and 12 months after cochlear implantation in deaf children $(\mathrm{N}=30)$.

\begin{tabular}{|l|l|l|l|}
\hline Acoustic parameter & Before $\mathbf{C l}$ (mean/S.D.) & $\mathbf{1 2}$ months after Cl (mean/S.D.) & p \\
\hline fO $(\mathrm{Hz})$ & $286.00 / 58.22$ & $274.46 / 75.31$ & 0.305 \\
\hline JIT $(\%)$ & $2.89 / 1.87$ & $1.52 / 1.25$ & $\mathbf{0 . 0 0 7}$ \\
\hline SH (\%) & $6.27 / 3.78$ & $4.14 / 1.98$ & $\mathbf{0 . 0 1 1}$ \\
\hline NHR & $0.18 / 0.11$ & $0.11 / 0.02$ & $\mathbf{0 . 0 1 0}$ \\
\hline
\end{tabular}

Bold values indicates statistical significance.

Table 3: Results of the acoustic analysis of the vowel /æ/ samples in children implanted before or at the age of 4 years $(\mathrm{N}=15)$ and children implanted after the age of 4 years $(\mathrm{N}=15)$.

\begin{tabular}{|l|l|l|l|}
\hline Acoustic parameter & $\begin{array}{l}\text { Implanted at the } \\
\text { Age }<\text { 4 years (mean/S.D.) }\end{array}$ & $\begin{array}{l}\text { Implanted at the } \\
\text { Age }>\text { 4 years (mean/S.D.) }\end{array}$ & 0.550 \\
\hline f0 before Cl & $303.73 / 75.69$ & $288.19 / 67.53$ & 0.018 \\
\hline f0 6 months after Cl & $317.77 / 43.07$ & $288.19 / 67.53$ & 0.008 \\
\hline f0 12 months after Cl & $311.85 / 70.08$ & $288.19 / 67.53$ & 0.112 \\
\hline f0 24 months after Cl & $315.07 / 49.69$ & $270.58 / 85.73$ & 0.000 \\
\hline JIT before Cl & $3.91 / 1.64$ & $1.17 / 0.62$ & 0.266 \\
\hline JIT 6 months after Cl & $2.01 / 1.47$ & $1.33 / 0.95$ & 0.439 \\
\hline JIT 12 months after Cl & $2.01 / 1.47$ & $1.33 / 1.11$ & 0.962 \\
\hline JIT "24 months" after Cl & $0.88 / 0.53$ & $0.89 / 0.54$ & 0.000 \\
\hline SH before Cl & $0.88 / 0.53$ & $3.49 / 1.24$ & 0.113 \\
\hline SH 6 months after Cl & $4.89 / 1.98$ & $3.65 / 1.25$ & 0.024 \\
\hline SH 12 months after Cl & $4.89 / 1.98$ & $3.24 / 1.43$ & 0.530 \\
\hline SH "24 months" after Cl & $3.23 / 1.51$ & $2.89 / 0.81$ & 0.530 \\
\hline NHR before Cl & $0.23 / 0.12$ & $0.11 / 0.02$ & 0.530 \\
\hline NHR 6 months after Cl & $0.23 / 0.29$ & $0.12 / 0.01$ & 0.188 \\
\hline NHR 12 months after Cl & $0.15 / 0.04$ & $0.13 / 0.04$ & 0.297 \\
\hline NHR "24 month" after Cl & $0.11 / 0.01$ & $0.11 / 0.02$ & \\
\hline
\end{tabular}

Table 4: Results of the acoustic analysis of the vowel /æ/ samples performed before 6 and 12, months after the implantation in children implanted before or at the age of 4 years $(\mathrm{N}=15)$.

\begin{tabular}{|l|l|l|l|}
\hline Acoustic parameter & Before CI (mean/S.D.) & $\begin{array}{l}\mathbf{6} \text { months after } \mathbf{C l} \\
\text { (mean/S.D./p) }\end{array}$ & $\begin{array}{l}\mathbf{1 2} \text { months after CI } \\
\text { (mean/S.D./p) }\end{array}$ \\
\hline fO (Hz) & $303.73 / 75.69$ & $317.77 / 43.07 / 0.178$ & $311.85 / 70.08 / 0.599$ \\
\hline JIT (\%) & $2.89 / 1.87$ & $2.01 / 1.47 / 0.003$ & $1.52 / 1.25$ \\
\hline SH (\%) & $6.27 / 3.78$ & $4.89 / 1.98 / 0.004$ & $4.14 / 1.98$ \\
\hline NHR & $0.18 / 0.11$ & $0.18 / 0.23$ & $0.11 / 0.01 / 0.021$ \\
\hline
\end{tabular}

Table 5: Results of the acoustic analysis of the vowel /æ/ samples performed before 6 and 12, months after the implantation in children implanted after the age of 4 years $(\mathrm{N}=15)$.

\begin{tabular}{|l|l|l|l|}
\hline Acoustic parameter & Before $\mathbf{C l}$ (mean/S.D.) & $\begin{array}{l}\mathbf{6} \text { months after } \mathbf{C l} \\
\text { (mean/S.D./p) }\end{array}$ & $\begin{array}{l}\mathbf{1 2} \text { months after } \mathbf{C l} \\
\text { (mean/S.D./p) }\end{array}$ \\
\hline fO $(\mathrm{Hz})$ & $289.96 / 71.26$ & $317.77 / 43.07 / 0.178$ & $311.85 / 70.08 / 0.599$ \\
\hline JIT (\%) & $1.34 / 0.78$ & $1.34 / 0.95 / 0.997$ & $1.33 / 1.11 / 0.458$ \\
\hline SH (\%) & $3.15 / 1.24$ & $3.65 / 1.25 / 0.402$ & $3.24 / 1.43 / 0.494$ \\
\hline NHR & $0.11 / 0.01$ & $0.12 / 0.01 / 0.269$ & $0.13 / 0.04 / 0.274$ \\
\hline
\end{tabular}


implanted after the age of 4 years, significantly lower mean values for JIT, SH and NHR were detected in the children implanted earlier. After the implantation, the only difference was detected in the $\mathrm{fO}$ and $\mathrm{SH}$ measured 12 months after the $\mathrm{Cl}$ (Table 3). After a comparison of the results of the acoustic analysis performed before, 6 , and 12 , months after the implantation in the children implanted before or at the age of 4 years, a significant improvement was found in JIT, SH and NHR (Table 4). After a comparison of the results of the acoustic analysis performed before 6 , and 12 , months after the implantation in the children implanted after the age of 4 years, a significant improvement was found only in fo and SH (Table 5).

\section{Discussion}

The results of the present study have confirmed that cochlear implantation enables auditory control of voice production and improves voice quality. The deaf children who were implanted before the age of 4 years improved control of their pitch and loudness during phonation hence, they improve their voice quality more quickly and to a greater extent than the children implanted after the age of 4 years. In our study, the acoustic analysis of voice samples showed that fo decreased no earlier than 12 months after the implantation. These results were not in accordance with other studies. In a great majority of the other studies on the influence of cochlear implantation on fo for deaf people, fo showed a significant decrease after the implantation $[12,14,16]$. Some authors detected a significant decrease in a minority of patients; nevertheless, a post-operative decrease of fo was generally observed for all patients [8]. Most of the investigators studied fo during spontaneous talk or during the reading of a standard text. In our study, 15 children were 4-year-old or younger at the time of the implantation and had not mastered reading. Therefore, we decided to analyze the vowel/æ/ which could be produced by every young Arabic speaking child. This could be the reason that the fo in our study did not significantly decrease, as it did in other studies. After the hearing control of phonation is gained it is expected that the pitch and amplitude variability would decrease. The results of our study confirmed this expectation. JIT and $\mathrm{SH}$ were significantly lower, as early as 6 months after the implantation. Twelve months after the implantation JIT and SH even improved and NHR also significantly improved. We believe that JIT and SH in a vowel sample /æ/ can be a good and early indicator of an improved auditory control of phonation after implantation in young children. When the results of the acoustic analyses in the children who were implanted before or at the age of 4 years were compared with the children implanted after the age of 4 years, significantly lower mean values for JIT, SH and NHR were detected in the older children before the implantation. It is possible that older children have better control of phonation due to a more mature and more precise neuromuscular control of the muscles involved in voice production. After the implantation the measured values of JIT, SH and NHR decreased in both groups. The only significant difference between the age groups appeared in the fo and SH measured 12 months after the implantation. The lower $\mathrm{fO}$ in the older group is logical with regard to the size of the larynx. The results of the study also indicate that the control of loudness is more effective in older children with a more mature neuro-muscular control of phonation. Therefore, the younger group needed more time to catch up with the older group with regard to $\mathrm{SH}$. We tried to find out which age group gained more after the implantation. When we compared the results of the acoustic analyses performed before 6 , and 12 months after the implantation a significant improvement in JIT and SH appeared as early as 6 months after the implantation in the children implanted at or before the age of 4 years. Twelve months after the implantation the NHR also significantly improved. In the group of children implanted after the age of 4 years the only significant improvement was detected in $\mathrm{fO}, 12$ months after the implantation, and in $\mathrm{SH}$, also 12 months after the implantation. The lowering of the fo was probably not only the result of auditory control but also the result of the growth of the larynx even to a minor extent. Since increased variability in pitch and loudness is a characteristic of voice [19], we believe that the improvement in JIT and SH in the older group was the result of implantation-acquired auditory control of phonation but that it was also influenced by the normal maturation changes in the group of older children.

The results of our study are in accordance with the survey of Seifert, et al. [16]. It is believed that prelingually deafened children who receive a cochlear implant before or at the age of 4 years attain a quicker and better auditory control over their voice and speech. In conclusion, the results of the present study have confirmed that cochlear implantation enables the auditory control of voice production, especially the moment-to-moment control of pitch and loud-ness. The results indicate that JIT and SH in a vowel sample /æ/ can be a good and early indicator of improved phonation control, even in young children. The deaf children who were implanted before the age of 4 years improved their voice control more quickly and to a greater extent than the children implanted after the age of 4 years. In any estimation of the $\mathrm{fO}$ changes after cochlear implantation the influence of the gradual maturation of voice in children must also be taken into consideration.

\section{Conclusion}

The results of the present study have confirmed that cochlear implantation enables auditory momentto-moment control of pitch and loudness. The determination of jitter and shimmer in the vowel Arabic $/ æ /$ sample proved to be a good and early indicator of 
improved phonation control, even in young children. The deaf children who were implanted before the age of 4 years improved their voice quality and control more quickly and to a greater extent than the children implanted after the age of 4 years.

\section{References}

1. AJ Yates (1963) Delayed auditory feedback. Psychol Bull 60: 213-232.

2. GM Siegel, HL Pick Jr (1974) Auditory feedback in the regulation of voice. J Acoust Soc Am 56: 1618-1624.

3. JL Elman (1981) Effects of frequency-shifted feedback on the pitch of vocal productions. J Acoust Soc Am 70: 45-50.

4. G Zimmermann, P Rettaliata (1981) Articulatory patterns of an adventitiously deaf speaker: Implications for the role of auditory information in speech production. $J$ Speech Hear Res 24: 169-178.

5. RS Waldstein (1990) Effect of post lingual deafness on speech production: Implication for the role of auditory feedback. J Acoust Soc Am 88: 2099-2114.

6. AA Angelocci, GA Kopp, A Holbrook (1964) The vowel formants of deaf and normal-hearing eleven-to-fourteenyear-old boys. J Speech Hear Dis 29: 156-160.

7. Nickerson, Raymond S (1975) Characteristics of the speech of deaf persons. Volta Rev 77: 342-362.

8. MB Higgins, EA McCleary, AE Carney, L Schulte (2003) Longitudinal changes in children's speech and voice physiology after cochlear implantation. Ear Hear 24: 48-70.

9. RB Monsen (1979) Acoustic qualities of phonation in young hearing- impaired children. J Speech Hear Res 22: 270288.

10. SB Leder, JB Spitzer, P Milner, C Flevaris-Phillips, JC Kirchner, et al. (1987) Voice intensity of prospective cochlear implant candidates and normal hearing adult males. Laryngoscope 97: 224-227.

11. V Ball, KT Ison (1984) Speech production with electro cochlear stimulation. Br J Audiol 18: 251-258.

12. SB Leder, JB Spitzer, $P$ Milner, C Flevaris Phillips, F Richardson, et al. (1986) Reacquisition of contrastive stress in an adventitiously deaf speakers using single-channel cochlear implant. J Acoust Soc Am 79: 1967-1974.

13. GF Smoorenburg, T Huiskamp, M Langereis, A Bosman (1994) Effects of cochlear implantation on voice quality and speech production. In: LJ Hochmair-Desoyer, ES Hochmair, Advances in Cochlear Implantation. Manz, Wien, 374-379.

14. J Hamzavi, W Deutch, WD Baumgartner, DM Denk, O Adunka (2000) Cochlear implantation and auditory feedback. Wien Klin Wochenschr 112: 515-518.

15. S Monini, G Banci, M Barbara, MT Argiro, R Filipo (1997) Clarion cochlear implant: Short-term effects on voice parameters. Am J Otol 18: 719-725.

16. E Seifert, M Oswald, U Bruns, M Vischer, M Kompis (2002) Changes of voice and articulation in children with cochlear implants. Int J Pediatr Otolaryngology 66: 115-123.

17. MA Svirsky, EA Tobey (1991) Effect of different types of auditory stimulation on vowel formant frequencies in multichannel implant users. J Acoust Soc Am 89: 2895-2904.

18. PH Dejonckere, $\mathrm{P}$ Bradley, $\mathrm{P}$ Clemente, $\mathrm{G}$ Cornut, L Crevier-Buchman (2001) A basic protocol for functional assessment of voice pathology, especially for investigating the efficacy of (phonosurgical) treatments and evaluating new assessment techniques-guideline elaborated by the Committee on Phoniatrics of the European Laryngological Society (ELS). Eur Arch Otorhinolaryngol 258: 77-82.

19. IH Boltezar, Burger ZR, M Zargi (1997) Instability of voice in adolescence: Pathologic condition or normal developmental variation? J Pediatr 130: 85-90. 